\title{
EDITORIAL
}

\section{Attitudinally correct nomenclature}

\author{
A C Cook, R H Anderson
}

For half a century, inappropriate terms have been used to describe the various parts of the heart in a clinical context. Does the cardiological community have the fortitude to correct these mistakes?

t is an undeniable fact that cardiac components are not always described according to the position they occupy within the body. This situation was initially drawn to our attention by Francisco Cosio. Thanks to his efforts, an attitudinally correct nomenclature was proposed to account for those features known to be of particular interest to those dealing with abnormal cardiac rhythms. ${ }^{1}$ The problem, however, is not confined to the electrophysiology laboratory. In this review, we will highlight other areas in which, currently, cardiac structures are not described appropriately with regard to the rest of the thoracic coordinates.

Thus, it is an accepted fact that all structures within the body are best described following the convention of the anatomical position (fig 1). Irrespective of the posture, or the location of the body, the subject is considered to be standing upright and facing the observer. Coordinates are then derived according to the three orthogonal planes. Orthogonal planes are at right angles to each other. For the body, two of these planes are in the long axis, while the third plane is transverse and in the short axis. The two planes in the long axis run from front to back, the sagittal plane, and from side to side, the coronal plane. Structures lying within different parts of the sagittal plane are then said to be anterior or posterior relative to each other, according to whether they are to the front or to the back of the body. Within the coronal, or frontal, plane, structures are described as being to the right or the left. Alternatively, within the frontal plane, structures can also be said to be medial or lateral relative to the central plane running from the top to the bottom. Along this central plane, described as having superior and inferior ends, there are any number of transverse planes which run at right angles relative to both long axis planes.

These conventional coordinates, and the adjectives which describe them, have withstood the rigours of centuries of use in accounting for the relations of the various parts of the human body. Generations of medical practitioners have been introduced to the conventions during their groundings in topographic anatomy.

Correspondence to: Professor R H Anderson Cardiac Unit, Institute of Child Health, 30 Guilford Street, London WCIN IEH, UK

r.anderson@ich.ucl.ac.uk appropriate $^{1}$ terms is the more important for clinicians, since the patient is always seen as an intact individual, even during cardiac surgical procedures. Thus, although usually investigated in the prone position, as in cardiac surgery, or echocardiographic, angiographic, or magnetic resonance interrogations, the parts of the heart should still be described relative to the standard anatomic position. The need for such attitudinally correct description has long been recognised. It was emphasised in the 1970s by practitioners such as McAlpine, ${ }^{2}$ and Walmsley and Watson. ${ }^{3}$ When producing an atlas in association with Becker, also in the late $1970 s,{ }^{4}$ one of us was at pains to endorse the importance of this concept, deriding the so-called "Valentine" approach to cardiac anatomy. In most instances, we did embrace the attitudinally correct orientation, and used it when preparing most of our figures. But, when describing and illustrating the cardiac short axis, we reverted to descriptions derived from the Valentine orientation. We then ourselves used the inappropriate terminology then employed by electrophysiologists. ${ }^{5}$ Thus, when describing the short axis of the heart as viewed from its ventricular aspect, we mistakenly labelled the pulmonary trunk as occupying an anterior position, and the sternocostal surface as being to the right (fig 2). As already discussed, the problems inherent in this approach were crystallised by Cosio many years later. He pointed out that the use of the "Valentine" approach resulted in the observer being forced to describe a catheter being advanced through the inferior caval vein towards the triangle of Koch as moving from posterior to anterior. In reality, as he emphasised, it is predominantly moving upwards in the body (fig 3). As also discussed, these discrepancies which existed in describing the electrophysiological coordinates of the atrioventricular junctions have now been corrected in a consensus document produced by combined European and US working groups. ${ }^{1}$ These problems implicit in the Valentine approach, however, impact on at least two other crucially important areas within the heart.

LOCATION OF THE CORONARY ARTERIES

The coronary arteries are the first branches of the aorta. Two such arteries arise from two of the three aortic sinuses of Valsalva, permitting the sinuses themselves appropriately to be designated as right coronary, left coronary, and non-coronary (fig 4). The Nomina Anatomica, however, currently accounts for these sinuses using the "Valentine" orientation, describing the heart as if standing on its apex, with its own long axes occupying the sagittal and coronal planes (fig 5). This is fundamentally wrong. The heart needs to be rotated through a right angle, and tilted notably, so as to
It would be thought, therefore, that all of these terms would properly be used in descriptions of the heart. Correct use of such attitudinally 


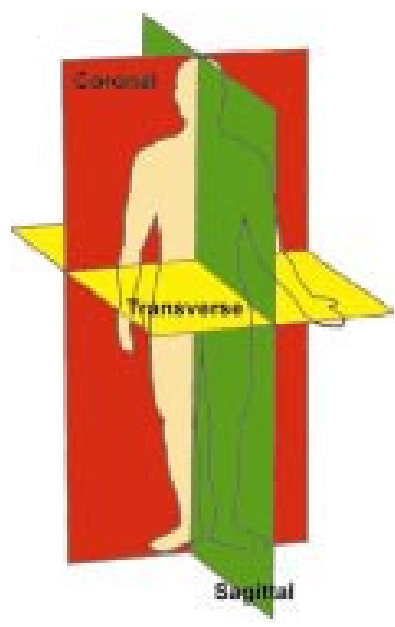

Figure 1 The subject is shown in the so-called anatomical position. Of the three orthogonal planes, the coronal plane extends from side-to-side, the sagittal plane from front to back, and the transverse planes run from top to bottom. Cardiac structure should be described within these coordinates rather than using the orthogonal planes of the heart itself.

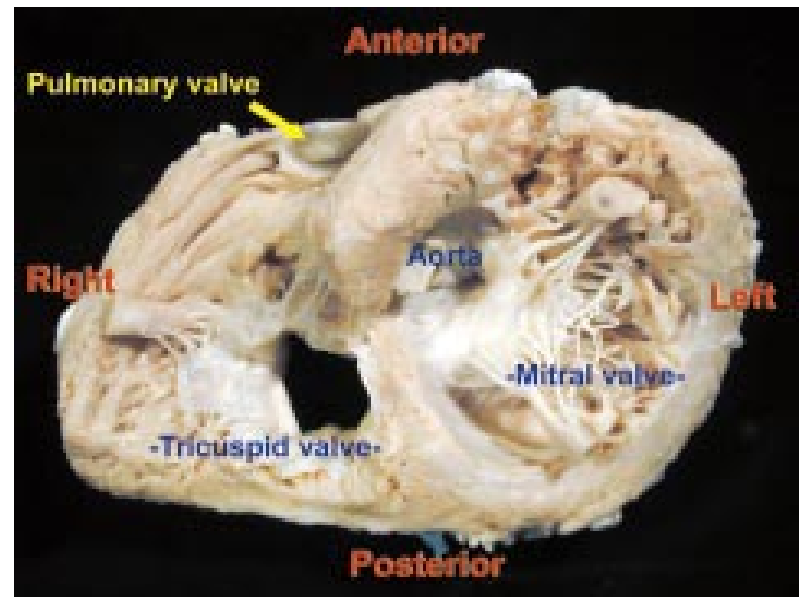

Figure 2 In the past, when describing the short axis of the heart as shown in this figure, seen from the ventricular aspect, we incorrectly labelled the superior aspect as "anterior", and the diaphragmatic surface as "posterior". This stems from the convention of placing the heart or its apex for description (see fig 5) rather than considering it as it lies within the body (see fig 6). The sternoscostal surface, shown as "right" in this picture, is more properly designated as being anterior (see fig 9).

be positioned in attitudinally correct orientation (fig 6). Examination of the heart as it lies properly within the chest then highlights the major deficiencies currently extant in the words used to describe the branches of the coronary arteries.

Thus, having emerged from the aortic sinuses, the major branches of the coronary arteries occupy either the atrioventricular or the interventricular grooves. The right coronary artery emerges directly into the right atrioventricular groove. It retains this position as it encircles the orifice of the tricuspid valve. In nine-tenths of individuals, said to have right coronary arterial dominance, this artery extends to the crux of the heart, where it gives rise to a major branch which occupies the interventricular groove of the diaphragmatic surface of the heart. Currently, this important artery is described as being "posterior" and "descending".

The left coronary artery emerges into the space between the pulmonary trunk and the left atrial appendage. It divides almost immediately into circumflex and so-called anterior descending branches. The circumflex artery then enters the left atrioventricular groove, with its extent being reciprocal to that of the right coronary artery. In one-tenth of individuals, it is the circumflex artery which reaches to the crux and gives rise to the artery which, as already explained, is said to be posterior and descending. This latter arrangement is known as

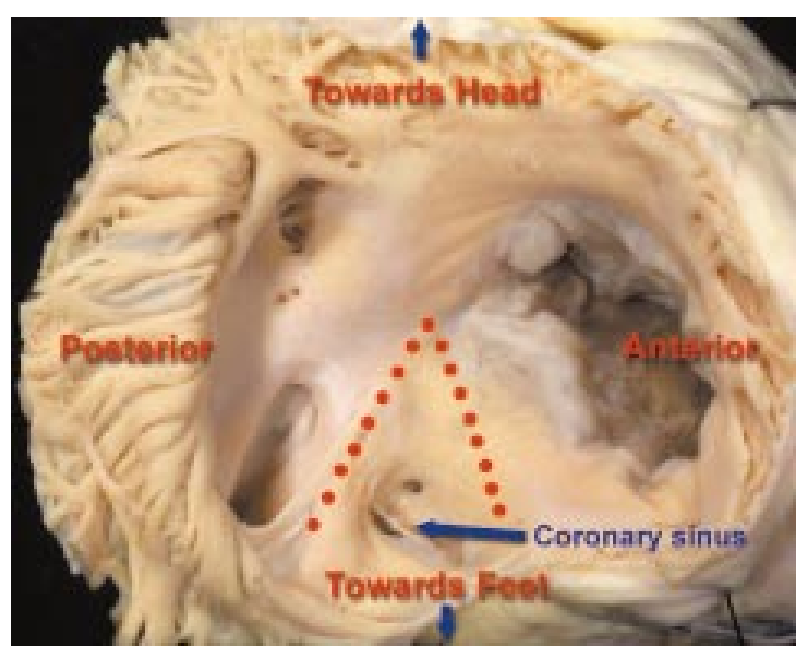

Figure 3 This shows the opened right atrium in attitudinally correct position. The dots delimit the triangle of Koch. When a catheter is advanced through the inferior caval vein towards the apex of the triangle, its tip moves towards the head, or superiorly. Using the incorrect convention shown in fig 2, it would be necessary to describe such a movement as extending anteriorly. In reality, to reach the apex of the triangle, the catheter would need to be moved superiorly and anteriorly.

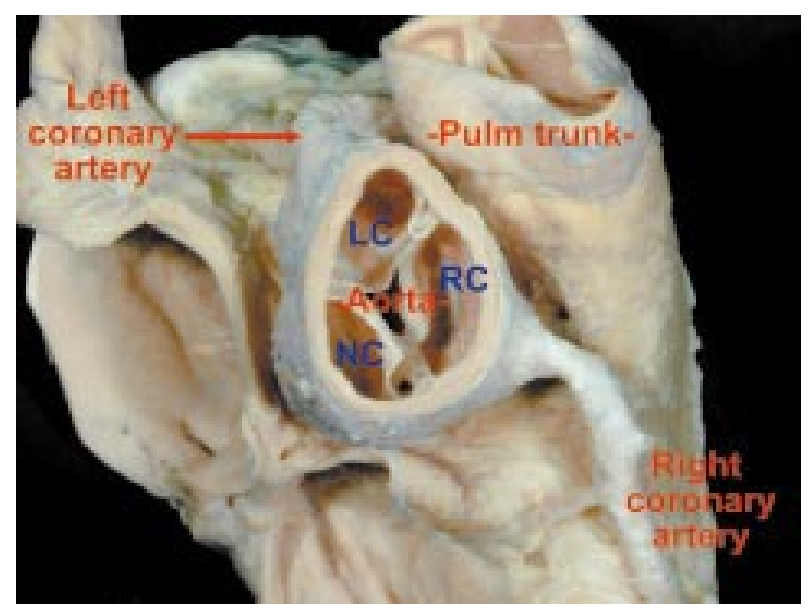

Figure 4 The base of the heart is shown from its atrial aspect, showing the central location of the aortic root. According to the origin of the coronary arteries, the aortic sinuses are best described as being left coronary $(\mathrm{LC})$, right coronary (RC), and non-coronary (NC).

left coronary arterial dominance. The other branch of the main stem of the left coronary artery occupies the interventricular groove that sits obliquely behind the left chest wall, runs down to reach the apex, and gives rise to its own septal perforating and diagonal branches. In current clinical parlance, this artery is described as anterior and descending.

The clinician is well aware that, in order to analyse the branches of the coronary arteries properly, it is necessary to use projections other than the frontal and lateral ones. Perhaps the most important projection is left anterior and oblique. Analysis of the cardiac short axis as seen in this plane (fig 7) then demonstrates the deficiencies with current nomenclature. When viewed in this oblique projection, it is an easy matter to appreciate the shape of the cardiac short axis, with its sternocostal, diaphragmatic, and pulmonary surfaces. When this arrangement is considered relative to the coordinates of the thorax, the diaphragmatic surface of the heart is seen to be positioned inferiorly (fig 8). A line drawn from top to bottom of this plane then divides the heart itself into anterior and posterior halves. When the heart is placed as close as 


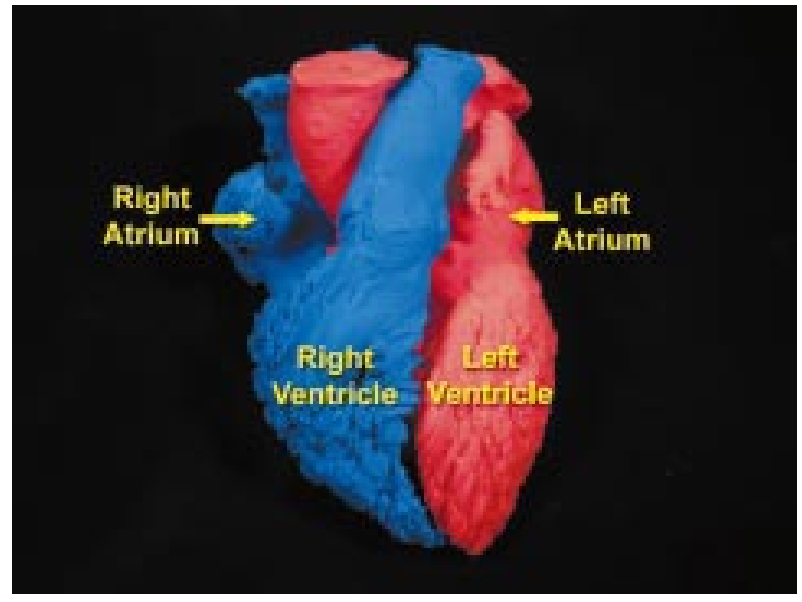

Figure 5 Using current nomenclature, the aortic sinuses are described as though the heart is positioned on its apex, as shown here. This orientation also accounts for the inappropriate nomenclature of the atrioventricular junctions as shown in fig 2 .

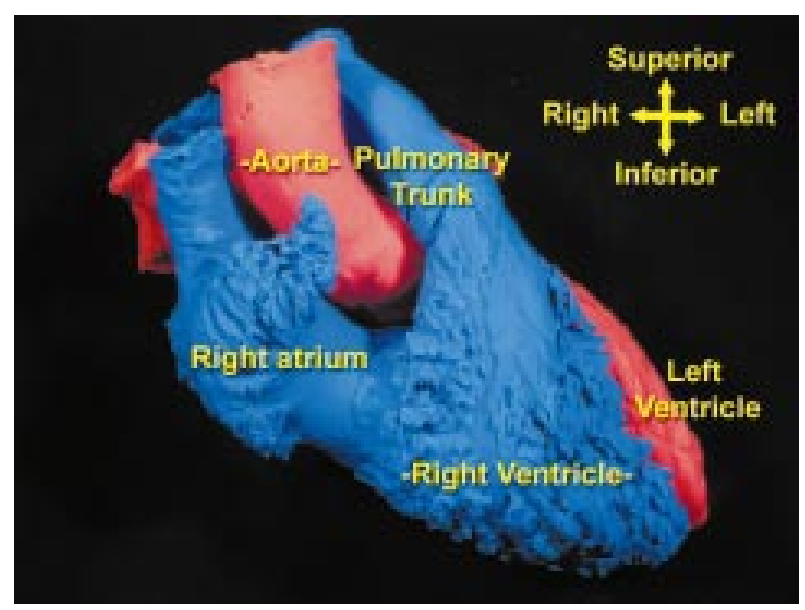

Figure 6 The cast shown in fig 5 has been repositioned and photographed in attitudinally correct orientation.

possible to the position it occupies during life, it is questionable whether the so-called "posterior" descending artery is truly posterior to its purportedly "anterior" partner (fig 8). It must also be questioned whether the artery descends as it runs from the crux to the apex of the ventricular mass. What is certain is that the area of musculature subtended by the artery is inferior rather than posterior, and that blockage of the artery supplying this area produces the electrocardiographic findings known to all as inferior myocardial infarction. Thus, if we used attitudinally correct nomenclature, the arteries running in the grooves between the ventricles would better be distinguished as the superior and inferior interventricular arteries.

\section{DESCRIPTION OF THE SEGMENTS OF THE VENTRICULAR MASS}

The left anterior oblique projection of the cardiac ventricular short axis (figs 7 and 9) is important not only to the coronary angiographer, but also to the nuclear cardiologist. When assessing the distribution of isotopes within the ventricular myocardium, it is customary to divide the short axis of the left ventricle into quarters (fig 8). At present, conventional wisdom dictates that it is the segment closest to the diaphragm which is called the "posterior" wall. This is incorrect. The mistake stems from the same difficulty as besets the electrophysiologist and the coronary angiographer, namely

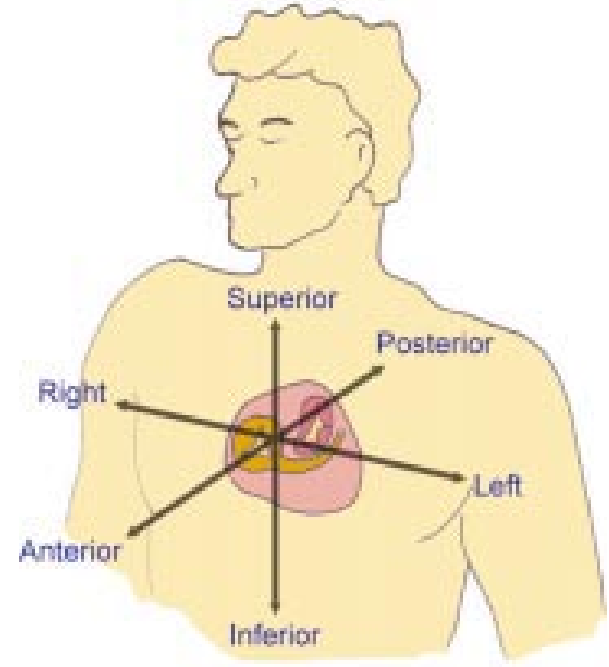

Figure 7 This diagram shows the atrioventricular junctions as seen in left anterior oblique orientation. The arrows show how the junctions relate to the orthogonal planes of the body. It is these bodily planes which should be used for anatomic description.

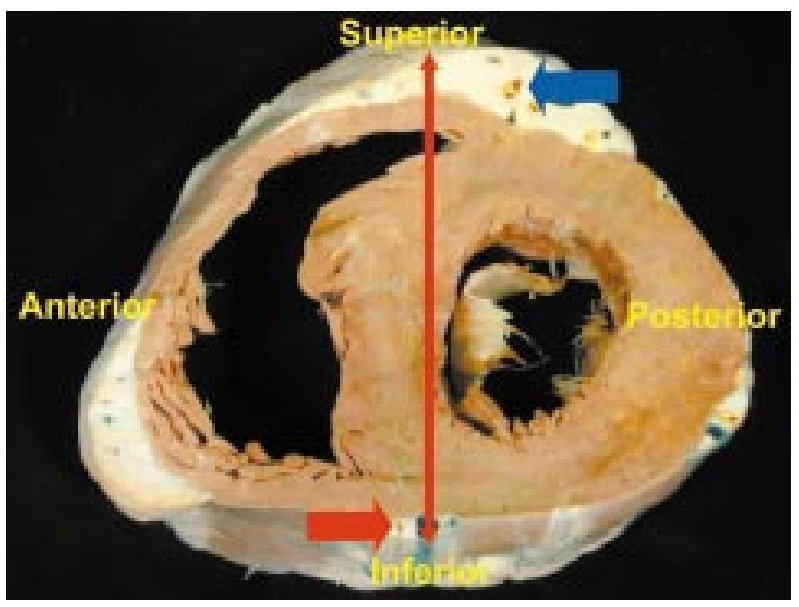

Figure 8 A short axis cut has been made through the ventricular mass replicating the left anterior oblique projection. Yellow injectate has been placed into the coronary arteries. Using current nomenclature, the artery shown supplying the top part of the muscular ventricular septum, and indicated by the blue arrow, is called the "anterior descending artery". As can be seen, in reality the artery is superior. It is, in fact, posterior to the artery supplying the diaphragmatic surface. This artery, shown by the red arrow, should properly be called the inferior interventricular artery.

the convention of describing the heart as though it was positioned upright on its ventricular apex. In reality, it is the segment of myocardium closest to the spine which is posteriorly located. The quadrant opposite to this, and hence anteriorly positioned, is made up mostly of the muscular septum. The other two quadrants, currently labelled "anterior" and "posterior", are positioned superiorly and inferiorly (fig 9).

\section{ATTITUDINALLY CORRECT NOMENCLATURE}

There continues to be a fundamental problem when the components of the heart are described in the clinical setting. This is because, traditionally, all the cardiac components have been named on the basis that the heart is positioned so that its own long axes are in the coronal and sagittal planes. All clinical cardiologists know intuitively that this is incorrect. Cosio and colleagues ${ }^{1}$ have already pointed to the advantages gained by 


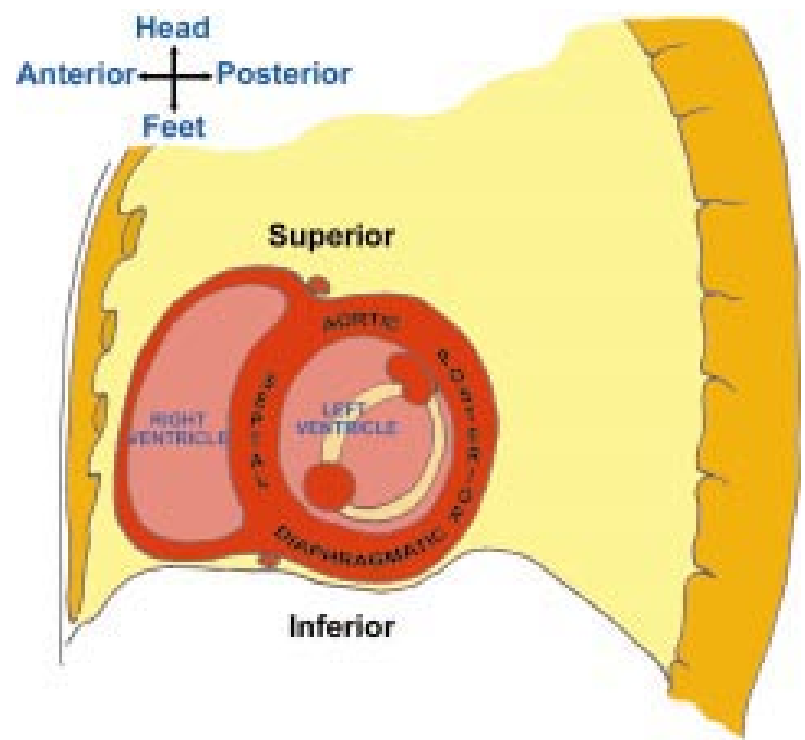

Figure 9 This diagram places the short axis of the ventricular mass, as shown in fig 8 , in the context of the thorax. As shown, the quadrants of the left ventricle as seen in this projection should properly be considered septal, aortic, posterior, and diaphragmatic At present, the inferior diaphragmatic surface is usually labelled, incorrectly, as being posterior.

the electrophysiologist when the heart is described using attitudinally appropriate nomenclature. ${ }^{23}$ When the triangle of Koch is depicted with its apex pointing to the top (fig 3), rather than to the right hand as has usually been the case, then the neophyte can understand that a catheter advanced through the inferior caval vein moves superiorly as well as anteriorly as it progresses to the apex of the triangle. It is very difficult for the uninitiated beginner to believe that upward motion of the catheter is turning its tip anteriorly. This description was mandatory when the coordinates of the cardiac short axis were described in the previously accepted electrophysiological fashion. Similar problems beset the tyro seeking to understand the interpretation of electrocardiographic evidences of myocardial ischaemia. Why is it that blockage of a supposedly "posterior" descending artery results in inferior myocardial infarction? Simply because the artery involved, in reality, is inferiorly positioned. And so it is with the quadrants of the left ventricular short axis. The true posterior quadrant of the left ventricle is the wall closest to the spine, while the anterior quadrant is the septal surface. The quadrant currently considered "posterior" is, in reality, the inferior wall of the left ventricle, lying as it does on the diaphragm.

If these problems are to be corrected, a sea change will be needed in the way the cardiologist describes the heart. Some excuse could be advanced to support the approach of the morphologist since, having usually removed the organ before its examination, it became customary to examine the organ with its own long axes occupying the sagittal and coronal planes. There is no such excuse for the echocardiographer, or angiographer, and even less for the resonance imager or nuclear cardiologist. With modern day techniques for imaging, the heart is always viewed in the context of its correct position within the body. With this information to hand, it should be an easy matter, for example, to appreciate that it is the quadrant of the ventricular circular short axis closest to the spine which is posterior. Similarly, the quadrant closest to the head is superior, and so on. We may all be surprised when we realise that, for half a century, we have used inappropriate terms to describe the various parts of the heart in a clinical context. The question now remains as to whether, as a cardiological community, we have the fortitude to correct our mistakes.

\section{ACKNOWLEDGEMENTS}

This work was supported by the British Heart Foundation together with the Joseph Levy Foundation. Research at the Institute of Child Health and Great Ormond Street Hospital for Children NHS Trust benefits from R\&D funding received from the NHS Executive.

\section{Authors' affiliations}

A C Cook, R H Anderson, Cardiac Unit, Institute of Child Health, University College, Great Ormond Street Hospital for Children Trust, London, UK

\section{REFERENCES}

1 Cosio FC, Anderson RH, Kuck K, et al. Living anatomy of the atrioventricular junctions. A guide to electrophysiological mapping. A consensus statement from the cardiac nomenclature study group, working group of arrhythmias, European Society of Cardiology, and the task force on cardiac nomenclature from NASPE. Circulation 1999; 100:e31-7

2 McAlpine WA. Heart and coronary arteries. An anatomical atlas for clinical diagnosis, radiological investigation, and surgical treatment. Berlin: Springer-Verlag, 1995

3 Walmsley R, Watson $\mathrm{H}$. Clinical anatomy of the heart. Edinburgh; Churchill Livingstone, 1978.

4 Anderson RH, Becker AE. Cardiac anatomy. Edinburgh; Churchill Livingstone, 1980

5 Sealy WC, Gallagher JJ, Wallace AG. The surgical treatment of the Wolff-Parkinson-White syndrome. Evolution of improved methods of identification and interruption of the Kent bundle. Ann Thorac Surg 1976;22:443-57. 